\title{
Evaluation of growth in children and adolescents after renal transplantation
}

\author{
Bahar Büyükkaragöz${ }^{1}$, Sevcan Azime Bakkaloğlu¹, Ayşe Fitnat Tuncel2, \\ Banu Kadıoğlu-Yılmaz ${ }^{3}$, Deniz Karcaaltıncaba ${ }^{4}$, Hatice Paşaoğlu ${ }^{2}$ \\ ${ }^{1}$ Division of Pediatric Nephrology, Departments of ${ }^{3}$ Pediatrics, ${ }^{2}$ Biochemistry and ${ }^{4}$ Perinatology, Gazi University Faculty \\ of Medicine, Ankara. E-mail: karamanbahar@yahoo.com \\ Received: 7th March 2017, Revised: 27th January 2018, 4th April 2018, 9th July 2018, Accepted: 18th \\ July 2018
}

\begin{abstract}
SUMMARY: Büyükkaragöz B, Bakkaloğlu SA, Tuncel AF, Kadığlu-Yılmaz B, Karcaaltıncaba D, Paşaoğlu H. Evaluation of growth in children and adolescents after renal transplantation. Turk J Pediatr 2019; 61: 217-227.

Despite the advances in the last decades, it is well-known that optimal growth is usually not achieved in children with chronic kidney disease (CKD) even after successful renal transplantation (RTx). In this study, our aim was to evaluate growth patterns and factors affecting growth in pediatric and adolescent renal transplant recipients (RTR). Thirty-seven prevalent RTR with mean age of $17.0 \pm 2.9$ years and mean post-RTx duration of $4.2 \pm 2.0$ years were evaluated. Growth parameters, height velocities and factors affecting growth at the time of RTx (baseline) and in the post-RTx follow-up were also retrospectively assessed. Cumulative corticosteroid (CS) doses were calculated. Mean height and weight standard deviation score (SDS) values were negative $(-1.4 \pm 1.1$ and $-1.2 \pm 1.5$, respectively), whereas height SDS was positive in $16 \%$ of the patients. Mean weight, height, and BMI (body mass index) SDS of the RTR were significantly higher than the values at transplantation $(\mathrm{p}<0.001$ for weight and height SDS; $\mathrm{p}<0.05$ for BMI SDS). Height SDS was <-2.0 in $19 \%$ of the patients while $60 \%$ at the baseline. Main factors associated with post-RTx height SDS were pre-RTx height SDS (B: $0.448, \mathrm{p}<0.01$ ) and CKD duration (B: $-0.01, \mathrm{p}<0.05)$. Although it was much better than the pre-RTx period, the present study reveals that postRTx growth was less than anticipated. As well as minimizing post-RTx CS doses and preserving graft function in the post-RTx follow-up, performing early transplantation and all efforts for minimizing pre-RTx growth deficit are crucial for an optimal post-RTx growth.
\end{abstract}

Key words: growth, adolescents, renal transplantation, body mass index, chronic kidney disease.

One of the ultimate goals of pediatric renal transplantation (RTx) is to maintain an optimal quality of life. Achieving a satisfactory final height is therefore crucial for normal psychosocial development which is an indicator of quality of care in children. ${ }^{1-2}$ Although RTx corrects many of the metabolic problems in the pre-RTx chronic kidney disease (CKD) period, post-RTx catch-up growth is generally insufficient to compensate for the pre-RTx growth deficit. ${ }^{3-4}$ Post-RTx growth is affected by three major factors: age at RTx, allograft function and corticosteroid (CS) dose. ${ }^{5}$ The degree of pre-RTx growth deficit, use of a deceased donor, presence of post-RTx persistent hyperparathyroidism and malnutrition are other negative predicting factors. ${ }^{3}$ Pubertal growth spurt is also delayed by 1.5 years and it lasts 1.6 years shorter in children with RTx; contributing to the persistence of growth retardation. ${ }^{1}$

It is well-known that resistance to growth hormone $(\mathrm{GH})$ is the main underlying mechanism of growth retardation in children with CKD. ${ }^{3-4}$ Randomized control studies have shown that recombinant human GH (rhGH) has a positive effect on growth and is safe in 
both children with CKD and those undergoing renal replacement therapy. ${ }^{6}$

In the last decades, with the interventions towards growth retardation, advances have been achieved in the growth of children in the CKD as well as in the post-RTx period. According to the North American Pediatric Renal Transplant Cooperative Study (NAPRTCS) 2010 report, height standard deviation scores (SDS) of children at the time of RTx were -2.43 in 1987; whereas this has improved over the years to -1.23 in the 2009 cohort. On the other hand, mean final height SDS of renal transplant recipients (RTR) over 19 years was reported to be -1.4 , whereas this number was still as low as -2.2 and -3.24 in $25 \%$ and $10 \%$ of those patients. Nevertheless, still far below than target final heights, there was an almost 1 SD improvement in adult height of RTR in the last 20 years; which emphasizes the importance of pre- and post-RTx interventions toward attaining optimal growth. ${ }^{7}$

Recent ESPN/ERA-EDTA registry showed that overweight and obesity, rather than underweight, are becoming prevalent in European children on renal replacement therapy. ${ }^{8}$ Children have a significant weight gain following RTx; especially in the post-RTx first year due to the use of high dose CS treatment. ${ }^{4,9}$ Rapid weight gain early after RTx is shown to be related to decreased graft survival. ${ }^{10-11}$ Although obesity is now been considered as an emerging problem among RTR $^{9,12-14}$, long term studies focusing on body mass index (BMI) in children with RTx are still scarce.

In the light of these data, we aimed to evaluate the growth and determine the relevant factors that would potentially affect growth patterns in children and adolescents with RTx in our center.

\section{Material and Methods}

This study was performed in February 2015 in 37 children and adolescents with RTx (13 girls/24 boys aged 11-21 years) followed at Gazi University Faculty of Medicine, Division of Pediatric Nephrology. Patients who received a transplant before the age of 18 years between January 2000 and January 2014, with at least one year follow-up were enrolled. For the patients who were transferred to the adult department, the data belonging to their last visits were recorded and included. The patients who were lost to follow-up or became dialysis dependent during their clinical course were excluded. The subjects or their parents have given their informed written consent and the study protocol was approved by the Research Ethics Committee of Gazi University Faculty of Medicine (13/04/2011-4002).

At the cross-sectional analysis, age, sex and anthropometric measurements (weight and height) of the RTR in February 2015 were recorded. This time point refers to the last visits of the patients who were included in the study, and therefore, might have consisted of different post-RTx times of the patients. Retrospectively, anthropometric measurements at RTx (baseline) and yearly after RTx as well as the clinical data including gestational age, birth weight, primary etiologies of end-stage renal disease (ESRD), duration of CKD, age at dialysis, pre-RTx dialysis modalities (peritoneal dialysis (PD)/hemodialysis (HD)), age at $\mathrm{RTx}$, donor type and post-RTx duration were recorded.

Weight and height SDS scores (z scores) were calculated using the formulas given below. SDS $=$ [observed value - median value of the reference population] / SD value of the reference population. ${ }^{14-16}$ SDS $<-2.0$ indicates scores below $3^{\text {rd }}$ percentile. BMI values of the RTR were calculated by using the formula of BMI = Weight $(\mathrm{kg}) /$ height $^{2}$ $\left(\mathrm{m}^{2}\right) .{ }^{13,17}$ BMI for age $<5^{\text {th }}$ percentile indicated undernutrition. ${ }^{18}$ BMI for age $>85^{\text {th }}$ and $<95^{\text {th }}$ percentile indicated overweightness, and $>95^{\text {th }}$ percentile indicated obesity. ${ }^{19}$ BMI SDS was expressed according to height-age. Height velocity SDS of RTR at different post-RTx time periods were also calculated. ${ }^{20-21}$

Pubertal status of the patient group was recorded using Tanner staging $22-23$ and the patients were classified as prepubertal, pubertal or postpubertal accordingly. Presence of pubertal delay was also noted. Pubertal status at RTx was obtained from patient charts.

None of the RTR was known to use rhGH therapy at the pre- and post-RTx period due to the legal restrictions. Immunosuppressive protocols in the post-RTx period were noted. Cumulative CS doses were calculated at the first, third and sixth month, first year, then one year apart, till February 2015. Graft functions 
Table I. Characteristics of the Study Group at the pre-RTx Period

\begin{tabular}{lcc}
\hline & $\mathrm{n}(\%)$ & Mean \pm SD \\
\hline Sex n Female & $13(35)$ & \\
$\quad$ Male & $24(65)$ & \\
Gestational age (weeks) & $38.5 \pm 1.2$ \\
Birth weight (kg) & $3.2 \pm 0.6$ \\
CKD duration (years) & & $4.3 \pm 2.5$ \\
Etiology of CKD & & \\
Congenital and genetic diseases & $18(49)$ \\
Vesicoureteral reflux & $10(27)$ \\
Posterior urethral valve & $3(8.1)$ \\
Autosomal recessive polycystic kidney disease & $1(2.7)$ \\
Bilateral multicystic kidney disease & $1(2.7)$ \\
Atrophic solitary kidney & $1(2.7)$ & \\
Cystinosis & $1(2.7)$ & \\
Juvenile nephronophthisis & $1(2.7)$ & \\
Acquired diseases & $8(22)$ & \\
Amyloidosis & $1(2.7)$ & \\
Pyelonephritis with recurrent nephrolithiasis & $1(2.7)$ & \\
Membranoproliferative glomerulonephritis & $1(2.7)$ & \\
Microscopic poliangitis & $1(2.7)$ & \\
Membranous glomerulonephritis & $1(2.7)$ & \\
Focal and segmental glomerulosclerosis & $3(8.1)$ & \\
Unknown diseases & $11(30)$ & \\
Age at ESRD & & $10.9 \pm 2.1$ \\
Age at dialysis onset & & \\
Duration of dialysis (years) & & \\
PD & & \\
HD & & \\
\hline
\end{tabular}

CKD, chronic kidney disease; ESRD, end stage renal disease; PD, peritoneal dialysis; $H D$, hemodialysis

and CKD stages were identified by using the creatinine levels of RTR. The glomerular filtration rates were calculated using the novel Schwartz formula. ${ }^{24}$ Patients with an increase in the serum creatinine concentration, which corresponds with a decrease in estimated glomerular filtration rate, were considered to have graft dysfunction. ${ }^{25}$

\section{Statistical analysis}

Differences between the two groups for continuous variables were evaluated by using Student's t- or Mann-Whitney U test and Kruskal-Wallis or ANOVA test, respectively, where applicable. Paired sample t test and Wilcoxon Sign Rank test were used in order to compare two related samples. Friedman test was used to compare three or more paired groups. Correlations between variables were evaluated by using Pearson and Spearman correlations, where applicable. Multiple linear regression analysis was performed to determine the variables that significantly affect post-RTx height SDS. SPSS version 17.0 was used (SPSS Inc., Chicago, IL, USA).

\section{Results}

\section{Pre-RTx period}

Majority of the patients were boys (65\%, $\mathrm{n}=24)$. Mean CKD duration was $4.3 \pm 2.5$ years. There were congenital and genetic causes of CKD in the half of the patients $(n=18$, $49 \%)$. Underlying disorders were unknown in $30 \%(n=11)$. Mean age at dialysis onset was $11.0 \pm 2.0$ years. Hemodialysis was the most common dialysis modality $(n=18,49 \%)$, and it was administered for a shorter duration than PD $(1.1 \pm 1.4$ vs $2.7 \pm 2.2$ years, $\mathrm{p}<0.01)$. Characteristics of the patient group at the preRTx period are given in Table I.

\section{Transplantation}

Mean age at RTx was $12.7 \pm 2.4$ years and did 
Table II. Anthropometric Measurements of the Study Group.

\begin{tabular}{lccc}
\hline $\begin{array}{l}\text { Patients } \\
(\mathrm{n}=37)\end{array}$ & $\begin{array}{c}\text { At the time of RTx } \\
\text { Mean } \pm \mathrm{SD}\end{array}$ & $\begin{array}{c}\text { Post-RTx value } \\
\text { Mean } \pm \mathrm{SD}\end{array}$ & $\mathrm{p}$ value \\
\hline Age (years) & $12.7 \pm 2.4$ & $17.0 \pm 2.9$ & \\
Weight (kg) & $32.8 \pm 9.9$ & $50.6 \pm 13.8$ & \\
Weight (SDS) & $-2.2 \pm 2.2$ & $-1.2 \pm 1.5$ & $\mathrm{p}<0.001$ \\
Height (cm) & $137.5 \pm 15.2$ & $156.2 \pm 11.7$ & \\
Height (SDS) & $-2.3 \pm 1.3$ & $-1.4 \pm 1.1$ & $\mathrm{p}<0.001$ \\
BMI & $17.1 \pm 2.0$ & $20.4 \pm 3.8$ & $\mathrm{p}<0.001$ \\
BMI (SDS) & $0.1 \pm 1.1$ & $0.4 \pm 1.1$ & $\mathrm{p}<0.05$ \\
\hline
\end{tabular}

$R T x$, renal transplantation; SD, standard deviation; SDS, standard deviation score; BMI, body mass index

not differ between gender $(12.3 \pm 2.3$ for girls vs $12.9 \pm 2.4$ for boys, $\mathrm{p}>0.05)$. None of the participants were infants or toddlers at the time of RTx. Preemptive RTx were performed in only 3 patients. Living-related RTx was applied in the majority $(n=26,70 \%)$. At this time, they were severely growth retarded (weight and height SDS as $-2.2 \pm 2.2$ and $-2.3 \pm 1.3$, respectively (Table II).

No differences were detected between PD and HD patients in terms of pre-RTx weight and height SDS ( $p>0.05$ for both, Table III). When the patient group was divided into two according to their ESRD etiologies, we observed that despite having lower values in patients with a congenital/genetic cause $(n=18)$, pre-RTx height and weight SDS were not significantly different from patients with acquired renal diseases $(n=8)$, as well $(p>0.05$ for both, Table III).

Body weight and height was below the 3rd percentile $(<-2 S D)$ in approximately $40 \%$ and $54 \%$ of the patients, respectively. Except one, all had both weight and height below 50th percentile with apparently greater height deficit (Fig. 1). At this era, mean height age was nearly 3 years below chronological age $(9.8 \pm 2.5$ years $)$. None of the children had BMI percentiles $<5$ th or $>95$ th indicating undernutrition or obesity (Fig. 2).

When the pubertal stages were evaluated separately in both genders, it was detected that $31 \%$ of the girls $(n=4)$ were prepubertal, $15 \%(n=2)$ were at puberty, $23 \%(n=3)$ were postpubertal and $31 \%(n=4)$ had pubertal delay at the time of RTx. At this period, $62 \%$ of the boys $(n=15)$ were prepubertal, $33 \%(n=8)$ were at puberty, $4 \%(n=1)$ had pubertal delay and none of them were postpubertal.

\section{Post-RTx period}

Mean age of the RTR was $17.0 \pm 2.9$ years with mean $4.2 \pm 2.0$ year post-RTx duration. Mean weight and height SDS of the patients were $-1.2 \pm 1.5$ and $-1.4 \pm 1.1$, respectively, indicating lower mean values than healthy peers (Table II). Nineteen\% $(n=7)$ still had values less than -2.0 SDS, whereas $16 \%$ of the RTR $(n=6)$ had positive height SDS. On the other hand, none of the patients had reached to a height SDS $>+2.0$.

Mean BMI SDS was in the normal ranges (Table II). However, $3 \%$ of the RTR $(n=1)$ had undernutrition, whereas $3 \%$ and $5 \%$ were overweight and obese, respectively $(n=1$ for overweight, $n=2$ for obese patients, Fig. 2).

It was observed that $50 \%$ of the boys $(n=12)$ and $85 \%$ of the girls $(n=11)$ were postpubertal.

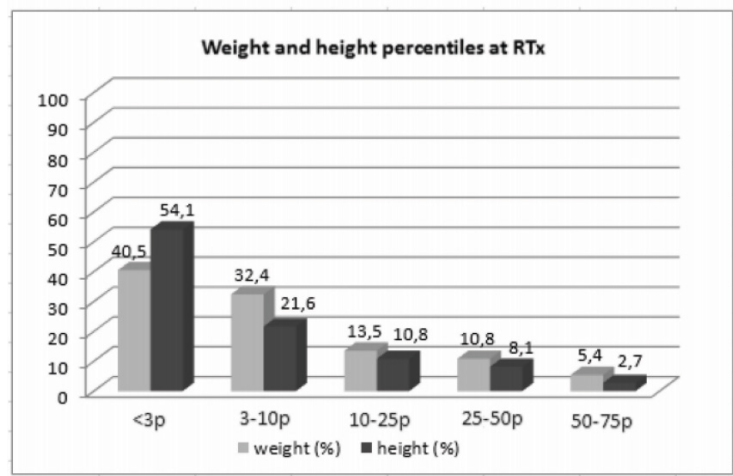

Fig. 1. Weight and height percentiles at RTx 


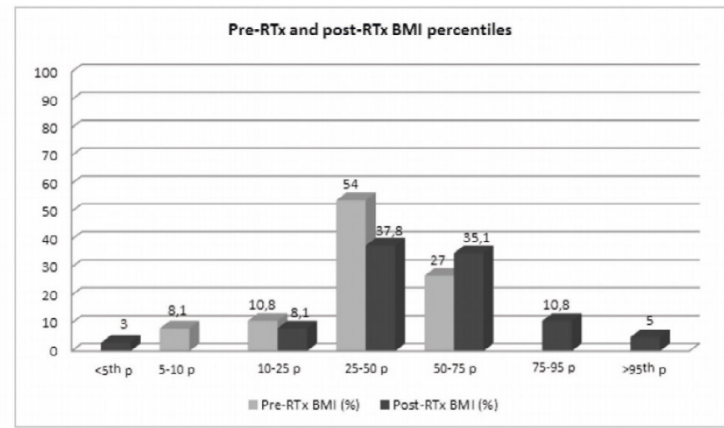

Fig. 2. Pre-RTx and post-RTx BMI percentiles

There were ongoing puberty in $33 \%$ of the boys $(n=8)$ and $8 \%$ of the girls $(n=1)$, while $17 \%$ of the boys $(n=4)$ and $8 \%$ of the girls $(n=1)$ were still prepubertal. None of the patients had pubertal delay.

The height and weight SDS of the patients were significantly higher than the values at transplantation $(p<0.001)$. Mean BMI SDS of the patients was also significantly higher than the baseline values, as well $(p<0.05$, Table II).

Post-RTx height SDS was not different in patients transplanted from either deceased or living donors $(-1.5 \pm 1.1$ vs $-1.4 \pm 1.1, \mathrm{p}>0.05)$. There were also no differences between the patients transplanted from deceased and living donors in terms of post-RTx weight and BMI SDS values ( $p>0.05$, for both). Similarly, postRTx weight and height SDS did not differ between either patients with a history of PD or HD administration or patients with acquired or congenital/genetic etiologies of ESRD ( $p>0.05$ for all, Table III).

Mean serum creatinine level and GFR was $1.4 \pm 0.8 \mathrm{mg} / \mathrm{dl}$ and $74.6 \pm 28.5 \mathrm{ml} / \mathrm{min} / 1.73$ $\mathrm{m}^{2}$, respectively. There were variable degrees of graft dysfunction in $65 \%$ of the RTR $(n=24)$. Although the RTR with graft dysfunction had slightly lower post-RTx SDS values $(-1.5 \pm 1.6)$ than the ones with well-functioning grafts $(-1.3 \pm 1.2)$, the difference was not statistically significant $(p>0.05)$. Similarly, there was no significant difference in respect to post-RTx weight SDS between the RTR with or without graft dysfunction ( $\mathrm{p}>0.05$, Table III).

\section{Changes in height SDS values and linear growth} patterns

All patients had negative height SDS at the baseline; $60 \%$ having values below -2.0 SDS 
Table IV. Changes in Height and BMI SDS at the Post-RTx Follow-Up.

\begin{tabular}{lcl}
\hline Patients & Height SDS & BMI SDS \\
\hline At RTx $(\mathrm{n}=37)$ & $-2.3 \pm 1.3$ & $0.1 \pm 1.1$ \\
Post-RTx sixth month & & $1.0 \pm 0.8$ \\
Post-RTx $1^{\text {st }}$ year $(\mathrm{n}=37)$ & $-2.1 \pm 1.2$ & $0.7 \pm 0.8$ \\
Post-RTx 2 $2^{\text {nd }}$ year $(\mathrm{n}=36)$ & $-1.8 \pm 1.2$ & $0.5 \pm 0.9$ \\
Post-RTx $3^{\text {rd }}$ year $(\mathrm{n}=31)$ & $-1.9 \pm 1.3$ & $0.5 \pm 0.4$ \\
Post-RTx 4 $4^{\text {th }}$ year $(\mathrm{n}=18)$ & $-1.9 \pm 1.5$ & $0.4 \pm 0.7$ \\
Post-RTx $5^{\text {th }}$ year $(\mathrm{n}=11)$ & $-1.4 \pm 1.5$ & $0.5 \pm 0.4$ \\
Post-RTx 6 $6^{\text {th }}$ year $(\mathrm{n}=6)$ & $-1.9 \pm 0.7$ & $0.4 \pm 0.6$ \\
Post-RTx $7^{\text {th }}$ year $(\mathrm{n}=5)$ & $-1.8 \pm 0.6$ & $0.4 \pm 0.4$ \\
Post-RTx $8^{\text {th }}$ year $(\mathrm{n}=2)$ & $-1.9 \pm 0.5$ & $0.5 \pm 0.2$ \\
\hline BML, body mass index; SDS, standard deviation score; RTx, renal transplantation &
\end{tabular}

BMI, body mass index; SDS, standard deviation score; $R T x$, renal transplantation

$(n=22)$ indicating severe growth retardation. Retrospective analysis of the post-RTx followup period revealed that although better than the values at transplantation, lowest values of height SDS were obtained at the post-RTx first year $(-2.1 \pm 1.2, \mathrm{n}=37)$. Thereafter, there was a gradual increase; with best scores obtained in the post-RTx fifth year $(-1.4 \pm 1.5)$. The yearly post-RTx height SDS values are given in Table IV and patterns of change in post-RTx height SDS are shown in Figure 3.

Mean height velocity SDS was positive throughout all post-RTx periods indicating a continuity of growth. On the other hand, the height velocity SDS was highest in post-RTx fifth year (Fig. 3).

\section{Changes in BMI SDS values}

Mean BMI SDS was highest in the post-RTx sixth month $(1.0 \pm 0.8)$. Thereafter, the values gradually decreased $(0.7 \pm 0.8$ and $0.5 \pm 0.9$ for post-RTx first and second year, respectively (Table IV).

\section{Immunosuppressive protocols}

Immunosuppressive protocol consisted of calcineurin inhibitors (cyclosporine $\mathrm{A}$ and tacrolimus), mycophenolate mofetil and CS combinations. Tacrolimus was the more commonly used calcineurin inhibitor (used in $68 \%$ of the RTR). Patients using cyclosporine A had significantly higher serum creatinine than those taking tacrolimus $(1.6 \pm 0.5$ vs $0.5 \pm 1.4, \mathrm{p}<0.05)$. Cyclosporine A was switched to tacrolimus in $16 \%$ of the cases late in the follow-up, and $5 \%$ of the patients $(n=2)$ were switched from tacrolimus to mTOR inhibitors (sirolimus) due to BK viremia. No differences were detected between patients using tacrolimus and sirolimus in terms of post- RTx weight and height SDS ( $\mathrm{p}>0.05$ for both, Table III).

Corticosteroids were initiated at high daily doses at the perioperative period, were gradually tapered after RTx, and minimized to $5 \mathrm{mg}$

Table V. Results of the Regression Analysis

\begin{tabular}{lccc}
\hline Variables of the modeling & $\mathrm{B}$ & $\mathrm{p}$ value & $\mathrm{SE}$ \\
\hline Model constant & 1.254 & & 1.001 \\
Pre-RTx height SDS & 0.448 & $\mathbf{0 . 0 0 2}$ & 0.130 \\
CKD duration & -0.010 & $\mathbf{0 . 0 4 1}$ & 0.005 \\
Age at RTx & -0.033 & 0.554 & 0.054 \\
Post-RTx duration & 0.001 & 0.448 & 0.005 \\
Creatinine level & -0.198 & 0.313 & 0.192 \\
Cumulative CS doses & -0.006 & 0.287 & 0.005 \\
\hline
\end{tabular}

RTX, renal transplantation; $C K D$, chronic kidney disease; SDS, standard deviation score; $C S$, corticosteroid 


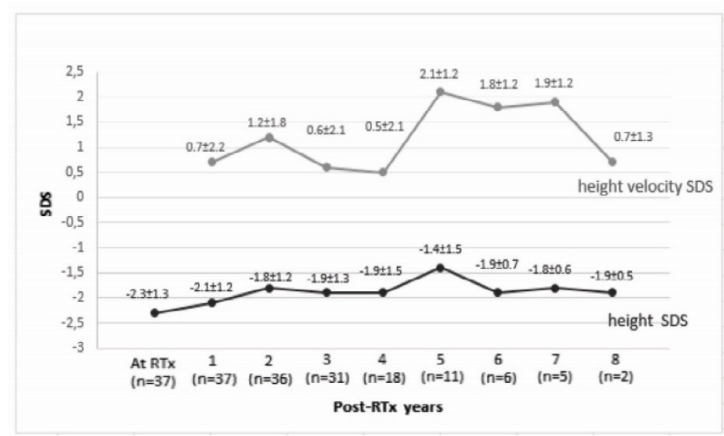

Fig. 3. Mean yearly changes in height and velocity SDS over post-RTx years

alternate daily after the early post-RTx period (which is three months post-RTx). Therefore, cumulative CS doses were highest in the early post-RTx period $(58 \pm 29 \mathrm{mg} / \mathrm{kg}$ and $75 \pm 42 \mathrm{mg} /$ $\mathrm{kg}$ at the first and third month, respectively). The doses diminished to $110 \pm 106 \mathrm{mg} / \mathrm{kg}$ at the end of the sixth month and $134 \pm 119 \mathrm{mg} /$ $\mathrm{kg}$ at the end of the first year. When calculated from the beginning, the cumulative CS doses reached to $170 \pm 124 \mathrm{mg} / \mathrm{kg}$ and $249 \pm 207 \mathrm{mg} /$ $\mathrm{kg}$ at the end of the second year, and at the end of third year and after, respectively. Therefore, despite apparent cumulative increase in CS dose over years, the rate of increase relatively diminishes after early post-RTx period due to low dose alternate day usage.

There was a strong negative correlation between the post-RTx height SDS and cumulative CS doses (first month: $\mathrm{r}=-0.552$, third month: $r=-0.540$, sixth month: $r=-0.529$, first year: $r=-0.519$, second year: $r=-0.552$, third year: $\mathrm{r}=-0.612, \mathrm{p}<0.01$ for all periods). In contrast, there was no correlation between the post-RTx height SDS and mean creatinine levels $(r=-$ 0.078, $\mathrm{p}>0.05)$.

There was a positive correlation between the post-RTx BMI SDS and cumulative CS doses at the third year and after $(\mathrm{r}=0.554, \mathrm{p}<0.05)$.

Multiple linear regression analysis revealed that pre-RTx height SDS and CKD duration were the most important parameters influencing post-RTx height SDS at the time of data collection (for pre-RTx height SDS; B value: $0.45, \mathrm{p}<0.01$ and for CKD duration; $\mathrm{B}$ value: $-0.01, \mathrm{p}<0.05)$ (Table V).

\section{Discussion}

Short stature still remains to be a great challenge which is difficult to manage and causes an important comorbidity in pediatric RTR. ${ }^{26}$ Growth failure is also a common feature in children with CKD and the final height of children with CKD is highly shorter than their healthy peers ${ }^{27}$. As previously mentioned, $\mathrm{GH}$ resistance is the most important factor in the etiology of growth retardation in CKD. Insufficient nutritional intake, CKD mineral and bone disorders, presence of comorbidities, metabolic acidosis, primary cause of renal disease, tubular disorders, pre-RTx use of CS treatment and anemia also contribute. $3,4,27,28$ In the present study, mean age at the baseline was $12.7 \pm 2.4$ years with pre-RTx CKD duration of more than 4 years. At that time, the patients were severely growth retarded and mean height age was approximately 3 years below the chronological age. As most of the children were referred to our center for RTx, we can not make a clear conclusion for the etiologies of their pre-RTx growth deficit. However, although statistically insignificant, height SDS of the patients with congenital and genetic diseases at RTx was lower than the cases with acquired diseases. Therefore, we believe that CKD etiology of the patients could be a contributing factor to their growth deficit. Besides, more than half of the patients had weight SDS less than -2 SD and a great proportion of the remainders had a weight percentile less than the 50th percentile at baseline. For this reason, although the specific etiological factors for undernutrition were not separately investigated in our study, we may speculate that loss of appetite due to uremia, severe dietary restrictions due to hyperphosphatemia and hyperkalemia, anemia and overt or subclinical inflammation could be considered as potential causes of undernutrition which played a role in their pre-RTx growth deficit. Lastly, regarding the fact that $\mathrm{rhGH}$ therapy is not routinely used for the children with CKD due to the financial issues in our country, we may consider this restriction itself as one of the major causative factors that contributed to this adverse outcome.

In this study, the main factors associated with post-RTx height SDS were pre-RTx height SDS and CKD duration. Both height and weight SDS 
of the transplanted children and adolescents were still significantly lower than the healthy peers. Although RTx was performed at relatively older ages with long pre-RTx CKD duration, the height SDS was positive in some patients and mean height SDS increased at a rate of $40 \%$ (from -2.3 to -1.4 ) compared to the pre-RTx era. Therefore, still having significantly lower height SDS than healthy children, RTx appeared to be beneficial in our patient group despite the aforementioned factors impairing growth.

Seven patients had height SDS $<-2.0$. Four of them were those transplanted older than 15 years of age with low height SDS values at the baseline and relatively short post-RTx follow-up. We believe that their height gain was limited due to exposure to high dose CS therapy and as a consequence of their low preRTx height SDS values. The remainders were those transplanted younger than 10 years of age with again a short follow-up period (mean 2 years). For this reason, although they had good graft functions, as the pubertal growth spurt did not occur and they were also exposed to high-dose CS therapy, their height gain was not as much as desired, as well.

Height velocity SDS was positive throughout all post-RTx periods indicating a persistence of linear growth despite the prolonged CS exposure or the presence of variable degrees of allograft dysfunction. Moreover, GH therapy was not administrated in any of the RTR during their clinical course. In the first four yearpost-RTx follow-up, growth velocity SDS was greater in the post-RTx second year compared to other time periods. In the present study, mean age at RTx was 12.8 years, with no difference between gender. Growth spurt in children is known to occur starting from Tanner stages 3-4, which is approximately 11.5 and 13.5 years in girls and boys, respectively ${ }^{29-31}$. Although Tanner staging was not routinely performed, as the present patients were on average 14 years old at post-RTx second year, we assume that this time with the higher slope of height velocity coincided with their growth spurt. On the other hand, there was a peak of growth velocity in the post-RTx fifth year. At that year growth velocity rates were noted in 8 patients, 6 of which were transplanted at ages less than 10 years. Therefore, we believe that this peak also coincided with the growth spurt in those children.

Corticosteroids impair growth by interfering with the growth hormone/insulin-like growth factor axis. In addition, they directly induce growth plate inhibition ${ }^{3}$. Switching from daily to alternate day use, CS withdrawal or avoidance all lead to improved growth velocity in RTR $^{5}$. In the present study, we demonstrated a significant negative correlation between mean height SDS and the cumulative CS doses at all post-RTx periods. However, regression analysis revealed that cumulative CS doses were not found to be a main predictor on post-RTx height SDS. Nevertheless, we believe that this data indicates the importance of the use of CS treatment as minimal as possible in order to ensure ideal growth in pediatric RTR.

Calcineurin inhibitors, despite not having direct effects, may indirectly deteriorate growth in RTR by causing nephrotoxicity and decreasing GFR ${ }^{32}$. Although immunosuppressive protocols were all based on calcineurin and CS combinations in the present study, we observed that RTR using cyclosporine A had higher creatinine levels. Therefore, we suggest that preference of tacrolimus would be a better strategy for preventing graft dysfunction. On the other hand, two patients were switched from tacrolimus to mTOR inhibitors due to BK viremia in the post-RTx follow-up. However, neither of these patients had elevation in the serum creatinine levels and they were successfully managed with reduction in immunosuppression and cidofovir treatment. Moreover, we did not observe any differences in respect to height SDS values between patients using MTOR and calcineurin inhibitors.

It is well-known that reduced GFR in RTR also has a negative influence on growth as uremia induces resistance to growth hormone. The coexisting metabolic acidosis also has additional effects $^{31}$. Tejani et al. ${ }^{33}$ showed that a $1 \mathrm{mg} /$ $\mathrm{dl}$ increase in serum creatinine was associated with a 0.17 decrease in height SDS in RTR. In our study, there was a variable degree of graft dysfunction in $65 \%$ of RTR. Nevertheless, we believe that the mean creatinine level $(1.4 \mathrm{mg} / \mathrm{dl}$ ) of the patients (who were on average 17 years of age) indicates a relatively well-preserved graft function. No significant correlation was detected between mean creatinine levels and post-RTx height SDS. 
Besides, although having slightly lower values in RTR with graft dysfunction, height SDS did not significantly differ in patients with or without graft dysfunction. As a result, we believe that the graft dysfunction may have relatively less prominent effects on mean height SDS than other factors impairing growth.

Donor type may lead to a difference on growth patterns. It was shown that children with a living related donor graft had better height SDS and greater growth velocity compared to those with a deceased donor graft in a five year follow-up. ${ }^{34}$ Most of the RTR had a living related donor in the present study. However, no difference was present in respect to post-RTx height, weight, and BMI SDS between adolescents transplanted either from a deceased or living related donor. Dialysis is also a major factor which impairs growth velocity and children with preemptive RTx have better post-RTx height SDS than those with a prior dialysis history. ${ }^{35}$ In the present study, we did not find any significant difference in terms of both pre- and post-RTx height SDS between the patients undergoing $\mathrm{HD}$ and PD. However, due to the limited number of preemptively transplanted cases, we could not make a clear conclusion on the beneficial effect of preemptive RTx on height gain.

As mentioned before, the early post-RTx period is usually associated with significant weight gain; especially due to high dose CS use. Vester et al. ${ }^{9}$ reported that there was a rapid increase in BMI SDS in the post-RTx third month, later BMI remained stable but did not decline to pre-RTx values. Similarly, in the present study, mean BMI SDS significantly increased in the post-RTx sixth month (attributed to high CS doses) and although it gradually decreased at the post-RTx consecutive years, mean BMI SDS of the patients was significantly higher than baseline values. This finding was attributed to two factors: first, weight gain is rather more than the suboptimal increase in height and secondly, inadequate nutritional intake in the CKD period is mostly corrected after RTx. In contrast to recent studies which emphasize the importance of obesity in children with renal replacement therapy ${ }^{8}$, the percentage of being overweight or obese was relatively low in our study. Due the hygienic concerns and with the fear of losing the graft, our patients are mainly fed with healthy home-cooked food prepared with diminished amounts of salt. The caregivers are greatly conscious about the nutritional issues and try to keep their children away from Western type fast-food diets. Although the feeding habits of the patients were not investigated in the current study, and these comments remain rather speculative, the relatively low prevalence of overweightness or obesity in post-RTx follow-up can be the result of these factors.

Three adolescents were obese and overweight. Despite the mean BMI SDS being positively correlated with cumulative CS doses, regarding the mean long post-RTx duration (which was beyond 4 years), we may conclude that the CS effects were minimalized and the overweightness and obesity in those patients was most probably linked to excessive nutrition. Meanwhile, only one of the patients had undernutrition. That patient was transplanted with the diagnosis of amyloidosis secondary to Familial Mediterranean fever (FMF). Despite being given medication, he had frequent FMF attacks together with adrenal insufficiency due to amyloid accumulation and an accompanying eating disorder.

The present study has some limitations. In our small patient population, there is a lack of information about Tanner stages in the postRTx follow-up and target heights of the RTR which resulted in difficulties concerning the definite interpretation of our results.

Our study showed that RTx has overt beneficial effects on both body weight and height compared to pre-RTx CKD period. For this reason, we believe that post-RTx care was much better due to better compliance to medications and follow-up visits which led to better management of patients compared to pre-RTx dialysis period. Nevertheless, growth of the RTR was far from ideal due to profound effects of pre-RTx growth deficit as well as performing RTx in older aged-patients with a long CKD duration. Therefore, no matter how successful the post-RTx follow-up was, our study revealed that the untoward effects of the pre-RTx CKD period on growth appeared to be the primary determinant of final growth in RTR.

In conclusion, as well as minimizing post-RTx CS doses and preserving graft function in the 
post-RTx follow-up, pre-RTx care including rational nutritional management and $\mathrm{GH}$ treatment are of vital importance in achieving better height velocity after RTx. Another important strategy should be decreasing the time on the RTx waiting list. Although our national RTx program has a new scoring system which gives a high priority to pediatric patients, low RTx rate should be increased by encouraging cadaveric donation.

\section{REFERENCES}

1. Nissel R, Brazda I, Feneberg R, et al. Effect of renal transplantation in childhood on longitudinal growth and adult height. Kidney Int 2004; 66: 792-800.

2. Ulinski T, Cochat P. Longitudinal growth in children following kidney transplantation: from conservative to pharmacological strategies. Pediatr Nephrol 2006; 21 : 903-909.

3. Harambat J, Cochat P. Growth after renal transplantation. Pediatr Nephrol 2009; 24: 1297-1306.

4. Derakhshan N, Derakhshan A, Fallahzadeh MH, et al. Evaluation of growth and body mass index in children following kidney transplantation. Saudi J Kidney Dis Transpl 2011; 22: 428-432.

5. Fine RN. Growth following solid organ transplantation in childhood. Clinics. (Sao Paulo) 2014; 69(Suppl 1): 3-7.

6. Cayir A, Kosan C. Growth hormone therapy in children with chronic renal failure. Eurasian J Med 2015; 47: 62-65.

7. NAPRTCS 2010 Annual Report. Available at https:// web.emmes.com/study/ped/annlrept/2010_Report.pdf.

8. Bontuis M, van Stralen KJ, Verrina E, et al. Underweight, overweight and obesity in paediatric dialysis and renal transplant patients. Nephrol Dial Transplant 2013; 28(Suppl 4): iv195-iv204.

9. Vester U, Schaefer A, Kranz B, et al. Development of growth and body mass index after pediatric renal transplantation. Pediatr Transplant 2005; 9: 445-449.

10. Boschetti SB, Nogueira PCK, Pereira AML, Fisberg M, Pestana JOM. Prevalence, risk factors, and consequences of overweight in children and adolescents who underwent renal transplantation--short- and mediumterm analysis. Pediatr Transplant 2013; 17: 41-47.

11. Mitsnefes MM, Khoury P, Mcenery PT. Body mass index and allograft function in pediatric renal transplantation. Pediatr Nephrol 2002; 17: 535-539.

12. Plumb LA, Pitcher D, Tse Y, Shield JP, Inward C, Sinha MD; British Association for Paediatric Nephrology. Longitudinal changes in body mass index following renal transplantation in UK children. Nephrol Dial Transplant 2014; 29: 196-203.

13. Mucha K, Foroncewicz B, Ryter M, et al. Weight gain in renal transplant recipients in a Polish single centre. Ann Transplant 2015; 20: 16-20.
14. Franke D, Thomas L, Steffens R, et al. Patterns of growth after kidney transplantation among children with ESRD. Clin J Am Soc Nephrol 2015; 10: 127-134.

15. World Health Organization. Physical status: the use and interpretation of anthropometry. Report of a WHO Expert Committee. Technical Report Series No. 854. Geneva: World Health Organization, 1995.

16. Neyzi O, Furman A. Bundak R, Gunoz H, Darendeliler F, Bas F. Growth references for Turkish children aged 6 to 18 years. Acta Paediatr 2006; 95: 1635-1641.

17. Haslam DW, James WP. Obesity. Lancet 2005; 366 1197-1209.

18. Wang Y, Chen HJ. Use of Percentiles and Z -Scores in Anthropometry. In: Preedy VR (eds): Handbook of Anthropometry: Physical Measures of Human Form in Health and Disease, Springer 2012: 29-49.

19. Barlow SE; Expert Committee. Expert committee recommendations regarding the prevention, assessment, and treatment of child and adolescent overweight and obesity: summary report. Pediatrics 2007; 120(Suppl 4): S164-S192.

20. Tanner JM, Davies PSW. Clinical longitudinal standards for height and height velocity for North American children. J Pediatr 1985; 107: 317-329.

21. Backeljauw PF, Tulsidas Dattani M, Cohen Pinchas, Rosenfeld RG. Disorders of growth hormone/insulinlike growth factor secretion and action. In: Sperling MA (eds): Pediatric Endocrinology, Elsevier Saunders 2014: 292-404.

22. Marshall WA, Tanner JM. Variations in pattern of pubertal changes in girls. Arch Dis Child 1969; 44: 291-303.

23. Marshall WA, Tanner JM. Variations in the pattern of pubertal changes in boys. Arch Dis Child 1970; 45: 13-23.

24. Schwartz GJ, Muñoz A, Schneider MF, et al. New equations to estimate GFR in children with CKD. J Am Soc Nephrol 2009: 20: 629-637.

25. Goldberg RJ, Weng FL, Kandula P. Acute and chronic allograft dysfunction in kidney transplant recipients. Med Clin North Am 2016; 100: 487-503.

26. Gil S, Vaiani E, Guercio G, et al. Effectiveness of rhGH treatment on final height of renal-transplant recipients in childhood. Pediatr Nephrol 2012; 27: 1005-1009.

27. Powell DR. Effects of renal failure on the growth hormone-insulin like growth factor axis. J Pediatr 1997; 131(1 Pt 2): S13-S16.

28. Mekahli D, Shaw V, Ledermann SE, Rees L. Long-term outcome of infants with severe chronic kidney disease. Clin J Am Soc Nephrol 2010; 5: 10-17.

29. Abbassi V. Growth and normal puberty. Pediatrics 1998; $102(2$ Pt 3): 507-511.

30. Tanner JM. Growth at adolescence, (2nd ed). Oxford, England, Blackwell Scientific Publications, 1962.

31. Needlman RD. Growth and Development. In: Behrman RE, Kliegman RM, Jenson HB (eds): Nelson Textbook of Pediatrics, W.B. Saunders Company, 2000: 23-61. 
32. Cochat P, Harambat J. Maximizing growth in children after renal transplantation. Transplantation 2009; 88: 1321-1322.

33. Tejani A, Fine R, Alexander S, Harmon W, Stablein D. Factors predictive of sustained growth in children after renal transplantation. The North American Pediatric Renal Transplant Cooperative Study. J Pediatr 1993; 122: $397-402$.
34. Pape L, Ehrich JH, Zivicnjak M, Offner G. Growth in children after kidney transplantation with living related donor graft or cadaveric graft. Lancet 2005; 366: 151-153.

35.Englund MS, Tydén G, Wikstad I, Berg UB. Growth impairment at renal transplantation-a determinant of growth and final height. Pediatr Transplant 2003; 7: 192-199. 